\title{
Serum Exosomal MiR-92b-5p as a Potential Biomarker for Acute Heart Failure Caused by Dilated Cardiomyopathy
}

\author{
Tao Wu Yichen Chen $^{\mathrm{b}}$ Yantao Du ${ }^{\mathrm{b}}$ Jin Tao ${ }^{\mathrm{c}}$ Zhong Zhou ${ }^{\mathrm{a}}$ Zhuo Yang ${ }^{\mathrm{a}}$ \\ aCardiovascular Department, the Affiliated Hospital of Medical School of Ningbo University, Ningbo, \\ ${ }^{b} N i n g b o$ Institute of Medical Science, Ningbo, 'Zhejiang Pharmaceutical College, Ningbo, Zhejiang, China
}

\section{Key Words}

Exosome $\cdot$ MiR-92b-5p $\cdot$ Biomarker $\cdot \mathrm{DCM} \cdot \mathrm{AHF}$

\begin{abstract}
Background/Aims: MicroRNAs (miRNAs) can be used as biomarkers for cardiovascular diseases, especially for heart failure. However, there are few reports on serum exosomal miRNA biomarkers in patients with acute heart failure (AHF) due to Dilated cardiomyopathy (DCM). Methods: We analyzed 3 different serum exosomal miRNAs (exo-miR-92b-5p, exomiR-192-5p, and exo-miR-320a) in 43 patients with DCM-AHF and 34 healthy volunteers as a control group (CG) by using exosome separation followed by a quantitative reverse-transcript PCR assay. Exosomes were identified by electron microscopy, NaNOZS-90, and western blot analyses (CD63 and Hsp70). Results: Serum exo-miR-92b-5p expression was increased in DCM-AHF patients compared to the CG (Mann-Whitney $U$-test: $P<0.001$ ). Exo-miR-92b-5p was positively related to age and some ultrasound data (Spearman's correlation: exo-miR$92 \mathrm{~b}-5 \mathrm{p}$ vs. age, $r=0.297, \mathrm{P}=0.014$; exo-miR-92b-5p vs. left atrial diameter, $r=0.431, \mathrm{P}$ $<0.001$; exo-miR-92b-5p vs. left ventricular diastolic diameter, $r=0.419, \mathrm{P}<0.001$; exomiR-92b-5p vs. left ventricular systolic diameter, $r=0.446, \mathrm{P}<0.001$ ). Exo-miR-92b-5p was also negatively related to other ultrasound data (Spearman's correlation: exo-miR-92b-5p vs. left ventricular fraction shortening, $r=-0.497, \mathrm{P}<0.001$; exo-miR-92b-5p vs. left ventricular ejection fraction, $r=-0.482, \mathrm{P}<0.001$ ). The discrimination of DCM-AHF patients from the CG by exo-miR-92b-5p was demonstrated by a receiver operating characteristic curve (exomiR-92b-5p: cutoff value $=0.0023$, area under the curve $=0.808, \mathrm{P}<0.001$, sensitivity $=$ $62.8 \%$, specificity $=85.3 \%$ ). Conclusion: Serum exo-miR-92b-5p is a potential biomarker for the diagnosis of DCM-AHF.

T. Wu and Y. Chen contributed equally to this work. 


\section{Introduction}

Dilated cardiomyopathy (DCM) is one of the most common forms of cardiomyopathy and is the main cause of heart failure (HF) [1]. Acute heart failure (AHF) is a serious pathophysiological state of DCM. Presently, the diagnosis of AHF due to DCM (DCM-AHF) is mainly based on ultrasound data and clinical biochemical indices, such as brain natriuretic peptide (BNP). Ultrasound data can be used to determine whether pathogenesis is mainly caused by DCM, whereas the change of BNP levels can help to estimate whether a patient with DCM is in the dangerous state of AHF. However, the data from ultrasound examination and clinical biochemical testing of BNP are neither specific nor cost-effective for the diagnosis of DCM-AHF. Therefore, novel clinical diagnostic biomarkers need to be developed for the benefit of patients with DCM-AHF.

The cardiovascular system has specific expression profiles (including RNAs and proteins) to maintain its normal function. For example, miR-34a reduces the shortening of telomeres, damage of the DNA response, and myocardial cell apoptosis by targeting protein phosphatase 1 regulatory subunit 10 [2], and miR-10a regulates vessel formation by targeting fms-related tyrosine kinase 1[3]. However, the expression profiles might change during cardiovascular disease (CVD) [4]. For example, miR-29 was decreased after acute myocardial infarction, which targets a cadre of mRNAs encoding proteins involved in fibrosis to promote the fibrotic response [5]. This was confirmed in another report in which miR$29 \mathrm{~b}$ was shown to target insulin-like growth factor 1 and phosphatidylinositol 3-kinase and contribute to muscle atrophy [6]. Microarray profiling also showed alterations in genes related to ion channels by comparing DCM patients with controls: sodium voltage-gated channel beta subunit 2 was increased, while potassium voltage-gated channel subfamily J member $5 / 8$, chloride intracellular channel $2 / 3$, and calcium voltage-gated channel auxiliary subunit B2/A1C were decreased [7].

Exosomes are endogenous nano-vesicles (size $30-100 \mathrm{~nm}$ ) that can carry biological information and may regulate the function of target cells $[8,9]$. The contents of exosomes could be proteins and nucleic acids (including DNAs and RNAs). Accordingly, researchers are passionate about identifying and analyzing the information delivered by exosomes.

MicroRNAs (miRNAs) are one of the molecules carried in exosomes [10,11]. Due to their small size and protection given by exosomes, miRNAs can escape from nuclease degradation in the serum or plasma. In addition, miRNAs have been extensively reported as biomarkers in many human CVDs, including DCM. For example, Gupta et al. demonstrated that the levels of miR-548 family members (miR-548c and miR-548i) in peripheral blood mononuclear cells were decreased in 44 patients with relatively stable chronic HF (CHF) compared with 48 non-failing controls [12]. In another study, miR-21 was found to be increased in patients with DCM, while miR-29 (miR-29a/29b/29c) and miR-133 (miR-133a/133b/133c) family members were decreased [13]. Meanwhile, miRNA biomarkers have also been identified in patients with HF. For example, miR-423-5p, miR-320, miR-22, and miR-92b were increased in patients with CHF [14], and miR-192, miR-194, and miR-34a were elevated in patients with HF caused by acute myocardial infarction (AMI) [15]. Besides, plasma miR-210 expression levels were significantly higher in patients with New York Heart Association class III and IV HF than in those with class II HF and controls [16]. Some reports have identified markers related to acute exhaustive exercise in patients with CHF. For example, Xu et al. reported that serum miR-21, miR-378, and miR-940 levels were significantly up-regulated immediately following acute exercise [17]. Despite these reports on serum or plasma miRNA biomarkers, to the best of our knowledge, there are few reports on miRNA biomarkers in patients with DCM-AHF, especially miRNA biomarkers from serum exosomes. In this study, we compared 3 different serum exosomal miRNAs (exo-miRNAs) between patients with DCM-AHF and an age- and sex-matched control group (CG). We aimed to analyze their potential use as biomarkers for DCM-AHF. 


\section{Cellular Physiology Cell Physiol Biochem 2018;46:1939-1950 \begin{tabular}{l|l} 
DOI: 10.1159/000489383 & $\begin{array}{l}\text { O } 2018 \text { The Author(s). Published by S. Karger AG, Basel } \\
\text { www.karger.com/cpb }\end{array}$
\end{tabular}}

Wu et al.: Exo-miR-92b-5p as a Biomarker for DCM-AHF

\section{Materials and Methods}

Inclusion and exclusion criteria of the patients and healthy volunteers

We prospectively recruited 43 DCM-AHF patients and 34 age- and sex-matched CG volunteers at the Affiliated Hospital of Ningbo University from March 2015 to April 2017. Patients with DCM were diagnosed using the 1995 WHO/ISFC criteria [18]. The diagnosis of AHF was based on the guidelines of the American Heart Association [19, 20]. Eligible patients with DCM-AHF were those with new-onset or acute decompensation CHF as the primary cause of admission. Exclusion criteria for all participants were pregnancy and known or treated malignancies.

The characteristics of the patients with DCM-AHF were as follows: average age 62 years (range, 28-86 years); 32 men and 11 women. Patients with any other diseases such as coronary heart disease, primary valvular heart diseases, congenital heart diseases, rheumatic diseases, and tumors were excluded. CG volunteers had no coronary or valvular diseases or no evidence of cardiomyopathy, as confirmed by echocardiography. The characteristics of the volunteers from the CG were as follows: average age 57 years (range, 21-83 years); 23 men and 11 women.

This study was approved by the Institutional Review Board of the Affiliated Hospital of Ningbo University, and all participants provided written informed consent.

Serum sample collection

Venous blood samples were obtained from the patients with DCM-AHF when they were admitted to hospital and from the volunteers at 07:00 before breakfast.

Serum samples were collected using pro-coagulation tubes, followed with standing for $2 \mathrm{~h}$ and centrifuging at $1660 \times \mathrm{g}$ for $10 \mathrm{~min}$ at room temperature. At least $800 \mu \mathrm{L}$ serum was collected from each sample and stored at $-80^{\circ} \mathrm{C}$.

\section{Exosome isolation and particle size identification}

Serum was centrifuged at $2000 \times g$ for $20 \mathrm{~min}$ in order to sediment debris. Subsequently, an exosome isolation reagent (RiboBio, Guangzhou, China) was added to the serum and incubated at $4^{\circ} \mathrm{C}$ for $30 \mathrm{~min}$. Exosomes were collected by centrifugation at $15000 \times \mathrm{g}$ for $2 \mathrm{~min}$ at $4^{\circ} \mathrm{C}$. The size and concentration of the exosomes were quantified by NaNOZS-90 (Malvern Instruments Ltd., Malvern, UK).

\section{Transmission electron microscopy}

The exosomes were resuspended in $30 \mu \mathrm{L}$ of $1 \times$ phosphate-buffered saline, and then $10 \mu \mathrm{L}$ of the sample was dropped onto a copper grid and left to subside for $1 \mathrm{~min}$. Filter paper was used to absorb the remaining fluid, followed by dropping $10 \mu \mathrm{L}$ uranyl acetate on the copper grid, which was left to subside for another $1 \mathrm{~min}$, again removing the remaining fluid using filter paper. The sample was air-dried for 10 min at room temperature and examined under a transmission electron microscope (H-7650; Hitachi, Tokyo, Japan) at $80 \mathrm{kV}$.

\section{Western blot assay}

Serum exosomes were lysed with RIPA lysis buffer and protein concentration was determined. The proteins $(20 \mu \mathrm{g})$ were electrophoresed by SDS-PAGE and transferred onto a PVDF membrane. The membrane was blocked for $2 \mathrm{~h}$ at room temperature with $5 \%$ non-fat milk. The membrane was incubated with primary antibodies (1:1000, anti-CD63 antibody [TS63], ab59479, Abcam, Cambridge, UK; 1:1000, anti-Hsp70 antibody, \#4876, CST, Danvers, MA, USA) overnight at $4^{\circ} \mathrm{C}$, followed by a secondary antibody $(1: 2000$, goat anti-mouse IgG, HRP-conjugated for CD63 and 1:3000, goat anti-rabbit IgG, HRP-conjugated for Hsp70) for another $1 \mathrm{~h}$. Protein expression was assessed using a LI-COR model 3600 (LI-COR, Durham, NC, USA).

\section{Quantitative reverse transcription-polymerase chain reaction}

miRNAs were extracted from blood (serum) using an miRNA Quick Extraction Kit (Bioteke, Beijing, China) according to the manufacturer's protocol. Exo-miRNAs were dissolved in $30 \mu \mathrm{L}$ RNase-free water, followed by an evaluation of their concentration and purity with an ultraviolet spectrophotometer. The expression levels of exo-miR-92b-5p, exo-miR-192-5p, exo-miR-320a were determined using a standard polyA quantitative reverse transcription PCR (RT-PCR) assay.

The sequences of the reverse transcription adaptor primer, forward primers for miR-92b-5p, miR-192$5 p$, miR-320a, internal reference gene forward primer for miR-451b [21], and universal inverse primer in 
Wu et al.: Exo-miR-92b-5p as a Biomarker for DCM-AHF

the regular polyA RT-PCR assay were as follows: reverse transcription adaptor primer, $5^{\prime}$-gcgag cacag aatta atacg actca ctata ggttt ttttt ttttv n-3'; miR-92b-5p PF, 5' -aggga cggga cgcgg tgcag tg-3'; miR-192-5p, 5' -atgac ctatg aattg acagc c-3'; miR-320a, $5^{\prime}$-aaaag ctggg ttgag agggc ga-3'; miR-451b PF, 5'-tagca agaga accat tacca tt-3'; and universal reverse primer, $5^{\prime}$-gcgag cacag aatta atacg ac- $3^{\prime}$.

The polyA reverse transcription conditions were $37^{\circ} \mathrm{C}$ for $1 \mathrm{~h}$ and $95^{\circ} \mathrm{C}$ for 5 min to tail polyA at the end of mature miRNAs, which was followed by the reverse transcription reaction: $42^{\circ} \mathrm{C}$ for 5 min and $85^{\circ} \mathrm{C}$ for $5 \mathrm{~min}$. The PCR cycling conditions were $95^{\circ} \mathrm{C}$ for $10 \mathrm{~min}$, followed by 40 cycles of $95^{\circ} \mathrm{C}$ for $20 \mathrm{~s}$ and $62^{\circ} \mathrm{C}$ for $35 \mathrm{~s}$, and the melt curve stage of the ABI Q5 standard SYBR Green procedure.

\section{Statistical analysis}

The SPSS 18.0 Mann-Whitney $U$-test was used to analyze the expression of serum exosome miRNAs between DCM-AHF patients and the CG. Spearman's correlation test was used to analyze the relationship between the expression level of exo-miR-92b-5p and the ultrasound data. Fisher's exact test, Pearson's chisquare test, Mann-Whitney $U$-test, and Student's $t$-test were used to analyze the characteristic features of the DCM-AHF patients and the CG. A receiver operating characteristic (ROC) curve was generated using the expression level of exo-miR-92b-5p to separate DCM-AHF patients from CG volunteers. All statistical tests were two-sided, and $\mathrm{P}<0.05$ was considered statistically significant.

\section{Results}

\section{Exosome sample identification}

The characteristics of exosomes were determined by particle-size analysis, transmission electron microscopy, and western blot analysis. Exosomes in the serum exhibited a doublelayer membrane and ranged in size from 30 to $150 \mathrm{~nm}$ (Fig. 1A and 1B). In addition, CD63 and Hsp70, which are protein markers of exosomes, were used to confirm the evaluation of exosomes by western blot assay (Fig. 1C).

Comparison of the clinical characteristics between the DCM-AHF patients and CG

The clinical characteristics of the DCM-AHF patients and CG are shown in Table 1.

The two groups were similar regarding sex, age, body mass index (BMI), smoking and drinking habits, and hypertension status. Predicted and trivial significant changes, reflecting the different nature of DCM-AHF versus CG were echocardiographic indexes (Mann-Whitney $U$-test, $\mathrm{P}<0.001$ ), including left ventricular diastolic diameter (LVDD), left ventricular systolic diameter (LVSD), left atrial diameter (LAD), left ventricular ejection fraction (LVEF), and left ventricular fraction shortening (LVFS). However, some confounding factors, such

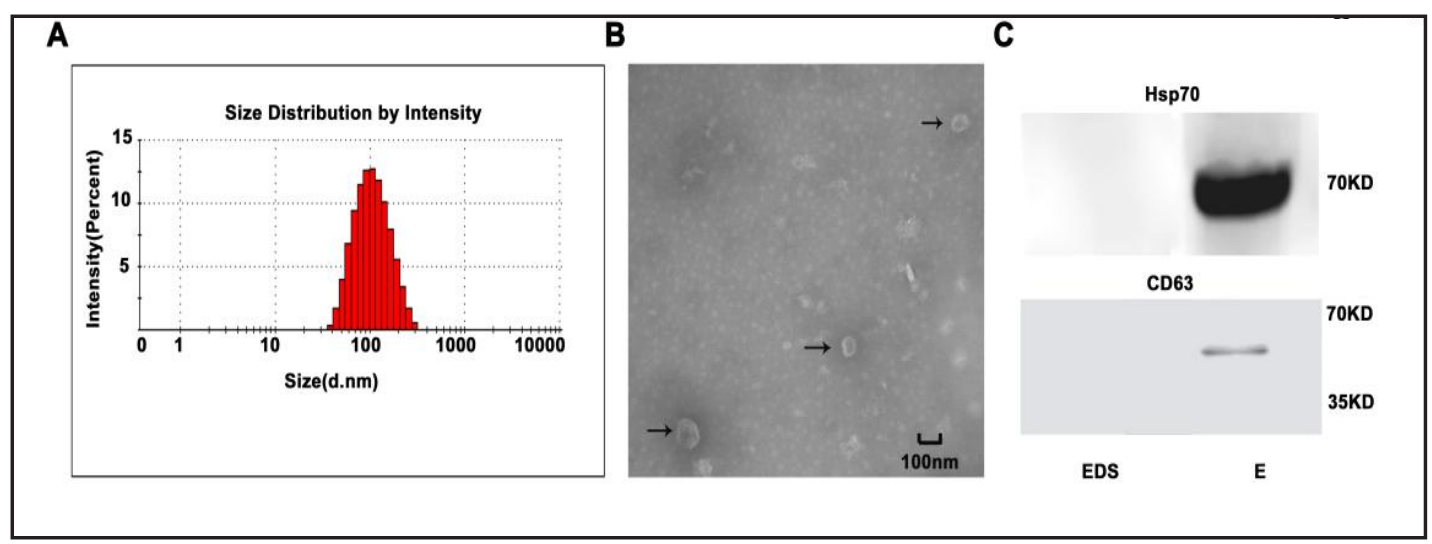

Fig. 1. Characterization of exosomes extracted from serum. A. The particle sizes of extracted exosomes were confirmed by NaNOZS-90. B. Serum exosomes were analyzed by microscopy, which were highlighted using black arrows. Scale bar $=100 \mathrm{~nm}$. C. The exosome biomarkers CD63 and Hsp70 were identified by western blot assay. E, exosomes; EDS: exosome-depleted supernatant. 
Wu et al.: Exo-miR-92b-5p as a Biomarker for DCM-AHF

as diabetes mellitus (Fisher's exact test, $\mathrm{P}=0.001$ ) and renal failure (Fisher's exact test, $\mathrm{P}=$ 0.009), also indicated significant differences between DCM-AHF and CG, which we analyzed further in the following sections.

\section{Exo-miR-92b-5p expression is increased in patients with DCM-AHF}

In this study, we pretested 9 serum exo-miRNAs in small groups and found that 3 different serum exo-miRNAs (i.e., exo-miR-92b-5p, exo-miR-192-5p, and exo-miR-320a) were relatively stably expressed (Table 2). Further formal tests showed that exo-miR-92b-5p was increased in DCM-AHF patients compared with the CG (Mann-Whitney $U$-test: $\mathrm{P}<0.001$; median [25-75\%]: DCM-AHF 0.0062 [0.0010-0.0119] vs. CG 0.0007 [0.0002-0.0016], Fig. $2 A)$. The expression of exo-miR-192-5p and exo-miR-320a was not significantly different between DCM-AHF and CG (Mann-Whitney $U$-test and median [25-75\%]: exo-miR-192-5p, $\mathrm{P}=0.454$, DCM-AHF 0.0028 [0.0010-0.0178] vs. CG 0.0035 [0.0010-0.0066], Fig. 2B; exomiR-320a, $\mathrm{P}=0.351$, DCM-AHF 0.0146 [0.0009-0.1398] vs. CG 0.0055 [0.0006-0.0705], Fig. 2C).

In order to characterize the main expression form of exo-miR-92b-5p in serum, we compared its expression between exosomes and exosomedepleted supernatant (Fig. 3), and found that miR-92b-5p was mainly expressed in exosomes.

In order to distinguish whether exomiR-92b-5p expression was affected by diabetes mellitus and renal failure, as mentioned above (Table 1), we further compared the expression of exo-miR$92 \mathrm{~b}-5 \mathrm{p}$ between DCM-AHF patients with and without diabetes mellitus as well as DCM-AHF patients with and without renal failure. There was no significant difference in exo-miR-92b-5p expression between DCM-AHF patients with diabetes mellitus and those without diabetes mellitus as well between DCM-AHF patients with renal failure and those

Table 1. Characteristics of the DCM-AHF and CG subjects a, Student t-test ; ${ }^{b}$, Pearson's Chi-square test; c, Fisher's exact test; ${ }^{d}$, Mann-Whiney $U$-test

\begin{tabular}{lccc}
\hline Variable & CG (n=34) & DCM (n=43) & P-value \\
\hline Age (years) & $57 \pm 2$ & $62 \pm 2$ & $0.107^{\mathrm{a}}$ \\
Sex (male) & $23(67.6 \%)$ & $32(74.4)$ & $0.514^{\mathrm{b}}$ \\
Smoking & $12(35.3 \%)$ & $20(46.5 \%)$ & $0.321^{\mathrm{b}}$ \\
Drinking & $8(23.5 \%)$ & $19(44.2 \%)$ & $0.059^{\mathrm{b}}$ \\
Diabetes mellitus & $4(11.8 \%)$ & $20(46.5 \%)$ & $0.001^{\mathrm{c}}$ \\
Hypertension & $22(64.7 \%)$ & $22(51.2 \%)$ & $0.233^{\mathrm{b}}$ \\
Renal failure & $1(2.9 \%)$ & $11(25.6 \%)$ & $0.009^{\mathrm{c}}$ \\
BMI (kg/m $)$ & $23.5 \pm 0.5$ & $24 \pm 0.6$ & $0.427^{\mathrm{a}}$ \\
BNP (ng/L) & $\mathrm{NA}$ & $927.8[637.4-1470.8]$ & $\mathrm{NA}^{\mathrm{a}}$ \\
LAD (mm) & $33.5[31.0-37.0]$ & $47.0[42.0-52.0]$ & $<0.001^{\mathrm{d}}$ \\
LVDD (mm) & $48.5[45.8-51.0]$ & $65.0[59.0-73.0]$ & $<0.001^{\mathrm{d}}$ \\
LVSD (mm) & $29.9 \pm 0.6$ & $52.3 \pm 1.1$ & $<0.001^{\mathrm{a}}$ \\
LVEF(\%) & $68.9 \pm 0.8$ & $39.1 \pm 1.4$ & $<0.001^{\mathrm{a}}$ \\
LVFS(\%) & $38.8 \pm 0.6$ & $20.3 \pm 0.8$ & $<0.001^{\mathrm{a}}$ \\
\hline
\end{tabular}
without renal failure (Fig. 4).
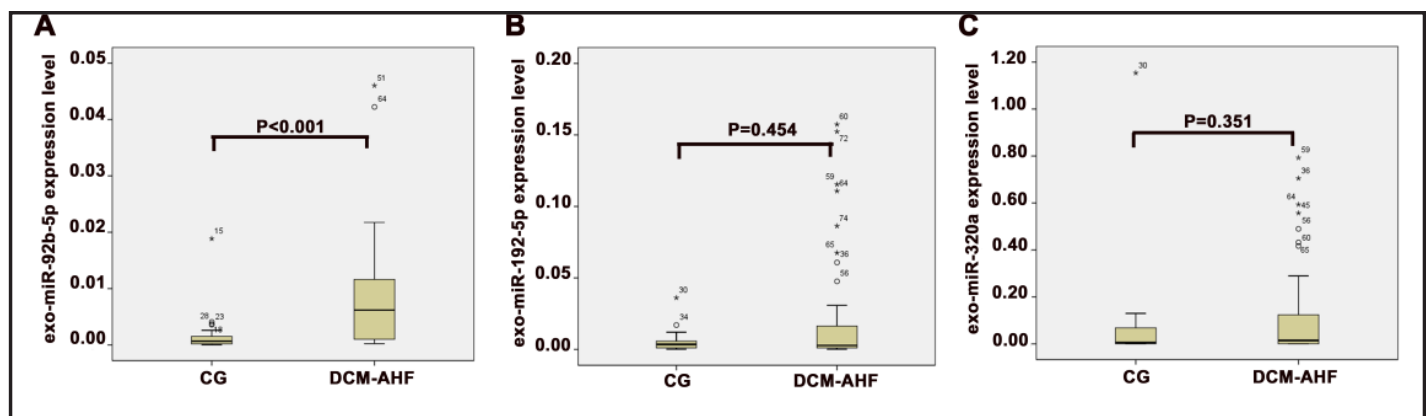

Fig. 2. Comparison of the expression levels of serum exo-miRNAs between DCM-AHF patients and the CG. A. Exo-miR-92b-5p (Mann-Whitney $U$-test: DCM-AHF vs. CG, P<0.001). B. Exo-miR-192-5p (Mann-Whitney $U$-test: DCM-AHF vs. CG, P = 0.454). C. Exo-miR-320a (Mann-Whitney $U$-test: DCM-AHF vs. CG, P = 0.351). 
Wu et al.: Exo-miR-92b-5p as a Biomarker for DCM-AHF

Fig. 3. Comparison of the expression levels of miR-92b-5p between exosomes and exosome-depleted supernatant. A. Relative expression levels of miR-92b-5p in exosomes and supernatant (exosome-depleted supernatant). B. Ratio of miR-92b-5p expression in exosomes and supernatant (exosomedepleted supernatant).

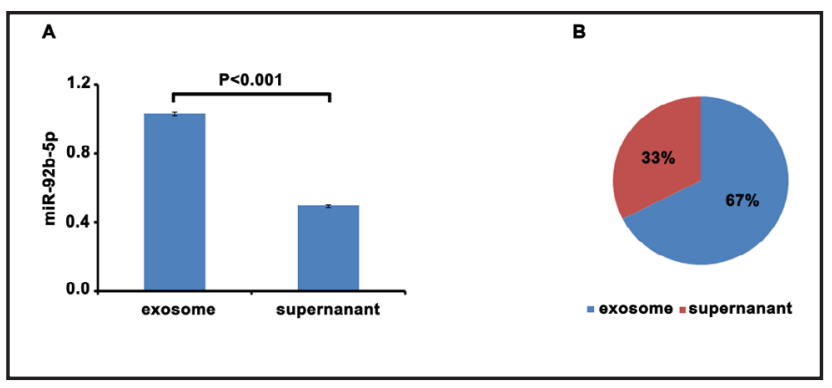

Fig. 4. Comparison of the expression levels of serum exo-miR-92b$5 p$ with clinical characteristics. A. Patients with or without diabetes mellitus. B. Patients with or without renal failure.

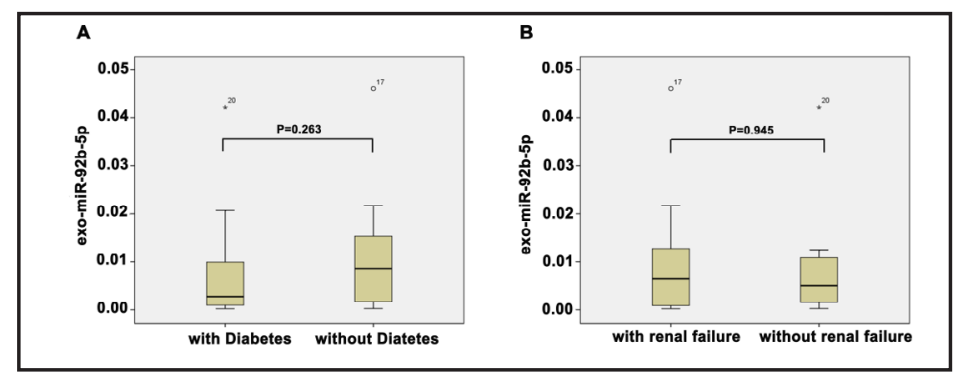

Relationship between the expression of exo-miR$92 b-5 p$ and other clinical characteristics

We further analyzed the relationship between exomiR-92b-5p and other clinical characteristics, and found that exo-miR-92b-5p was significantly related to age and echocardiographic indexes (Fig. 5). Exo-miR-92b-5p expression was positively related to LAD (Fig. 5A, Spearman's correlation, $r=0.431, \mathrm{P}<0.001$ ), LVDD (Fig. 5C, Spearman's correlation, $r$ $=0.419, \mathrm{P}<0.001$ ), LVSD (Fig. 5D, Spearman's correlation, $r=$ $0.446, \mathrm{P}<0.001$ ), and age (Fig. $5 \mathrm{~F}$, Spearman's correlation, $r=$ $0.297, \mathrm{P}=0.014$ ), whereas it was negatively related to LVEF (Fig. 5B Spearman's correlation, $r=-0.482$, $\mathrm{P}<0.001$ ) and LVFS (Fig. 5E, Spearman's correlation, $r=-0.497$, $\mathrm{P}<0.001$ ).
Table 2. Choice of candidate exo-miRNAs and references. Note:"-“= unrecorded

\begin{tabular}{|c|c|c|c|}
\hline Candidate Targets & & Pretest non-detection rate & Range of CT \\
\hline \multirow[t]{9}{*}{ miRNAs } & miR-1-2 & $7 / 15(47 \%)$ & - \\
\hline & miR-210 & $3 / 5(60 \%)$ & - \\
\hline & miR-499a & $4 / 5(80 \%)$ & - \\
\hline & miR-499b & $4 / 5(80 \%)$ & - \\
\hline & miR-208b & $13 / 15(87 \%)$ & - \\
\hline & $\operatorname{miR}-423$ & $11 / 15(73 \%)$ & - \\
\hline & miR-320a & $0 / 10(0)$ & - \\
\hline & miR-192-5p & $0 / 10(0)$ & - \\
\hline & miR-92b-5p & $0 / 10(0)$ & - \\
\hline \multirow[t]{5}{*}{ References } & U6 & $1 / 9(11 \%)$ & $28-31$ \\
\hline & miR-484 & $0 / 6(0)$ & $25-34$ \\
\hline & miR-16 & $7 / 12(58 \%)$ & $26-39$ \\
\hline & miR-642-3p & $0 / 6(0)$ & $28-32$ \\
\hline & miR-451b-5p & $0 / 6(0)$ & $22-24$ \\
\hline
\end{tabular}

\section{Exo-miR-92b-5p is a potential diagnostic biomarker for DCM-AHF}

The discrimination of DCM-AHF patients from CG volunteers by using exo-miR-92b$5 p$ was demonstrated by a ROC curve with an area under the curve (AUC) of 0.808 (95\% confidence interval, $0.712-0.903, \mathrm{P}<0.001$ ) (Fig. 6). When the expression value of exo-miR$92 \mathrm{~b}-5 \mathrm{p}$ was equal to or greater than 0.0023 , which predicts that patients have DCM-AHF, a sensitivity of $62.8 \%$ and specificity of $85.3 \%$ were generated for the identification of DCMAHF. 


\section{Discussion}

Circulating miRNAs are potential biomarkers for diverse CVDs [22, 23]. In the present study, we aimed to identify potential serum exo-miRNAs as diagnostic biomarkers for DCM-AHF. We found that serum exo-miR-92b-5p was significantly increased in patients with DCMAHF. Further analysis also indicated that serum exomiR-92b-5p is a potential biomarker for DCM-AHF. However, some issues require further discussion.

It is known that miRNAs are potential biomarkers for various diseases, because of their stability in human plasma or serum. Mitchell et al. first reported such a phenomenon in human prostate cancer [24]. However, as further research has been conducted, the stability of miRNAs has been found not only to be attributed to their small size but also to their protection in exosomes. Circulating miRNAs exist as 2 forms, namely, free miRNAs and exo-miRNAs. Gall et al. confirmed that there are differences between serum free nucleic acids and exosome-contained nucleic acids [25]. They reported that the majority of miRNAs were contained in exosomes in serum [25]. miRNAs, which are extracted from isolated exosomes, can be tested more sensitively than those in whole serum [25]. Some reports have supported this view. For example, Jansen et al. compared the expression of miRNAs between plasma and circulating microvesicles in 181 patients with stable coronary artery diseases, and found that miR-126 and miR-199a, which were contained in microvesicles, were related to cardiovascular events, but not the free nucleic acids circulating in the plasma [26]. Certainly, there has been some debate over this issue. For example, Goren et al. compared the expression of circulating miRNAs between

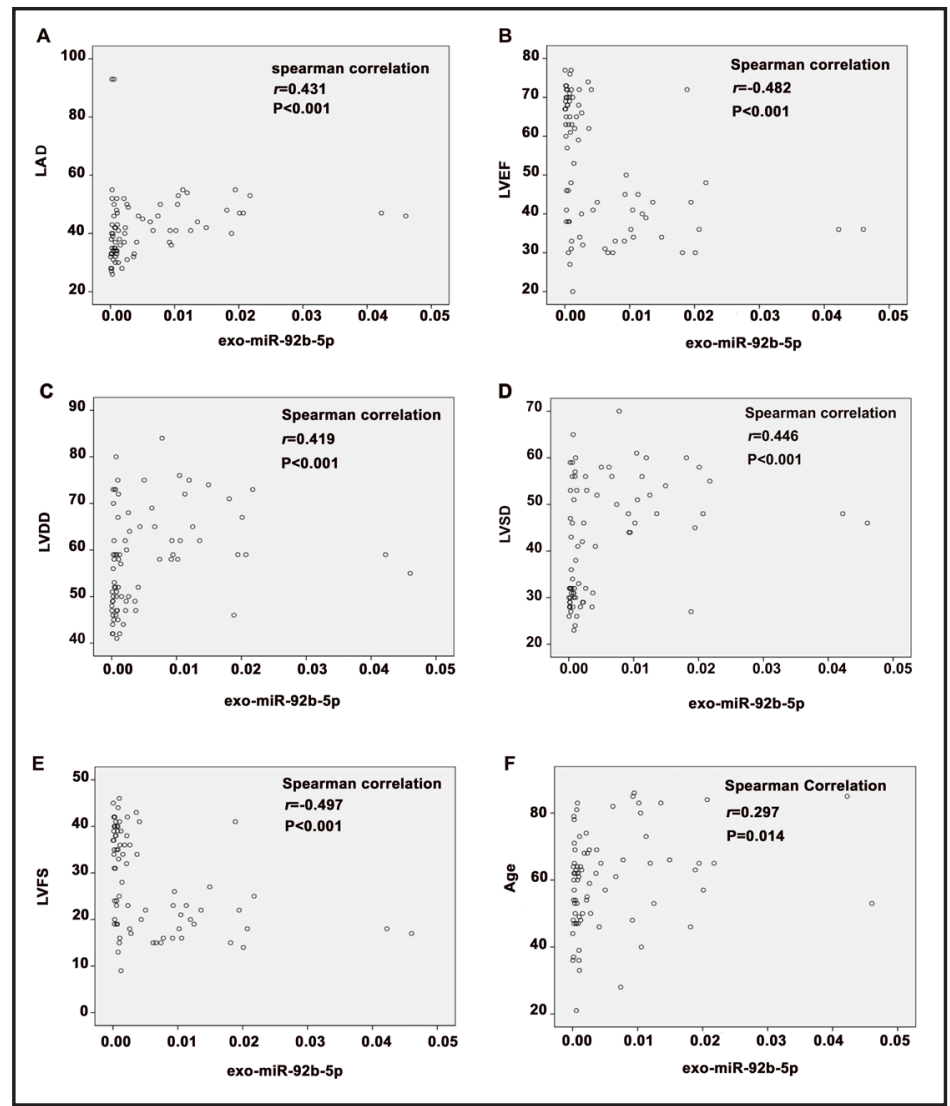

Fig. 5. The relationship between exo-miR-92b-5p and other clinical characteristics. A. exo-miR-92b-5p vs. LAD (Spearman's correlation: $r=$ 0.431, $\mathrm{P}<0.001$ ). B. Exo-miR-92b-5p vs. LVEF (Spearman's correlation: $r=-0.482, P<0.001)$. C. Exo-miR-92b-5p vs. LVDD (Spearman's correlation: $r=0.419, \mathrm{P}<0.001)$. D. Exo-miR-92b-5p vs. LVSD (Spearman's correlation: $r=0.446, \mathrm{P}<0.001)$. E. Exo-miR-92b-5p vs. LVFS (Spearman's correlation: $r=-0.497, \mathrm{P}<0.001$ ). F. Exo-miR-92b-5p vs. age (Spearman's correlation: $\mathrm{r}=0.297, \mathrm{P}=0.014$ ).

Fig. 6. ROC curve for discriminating DCM-AHF patients from the CG by exo-miR-92b5p. AUC: 0.859; cutoff value: 0.0023; sensitivity: 62.8\%; specificity: $85.3 \%$.

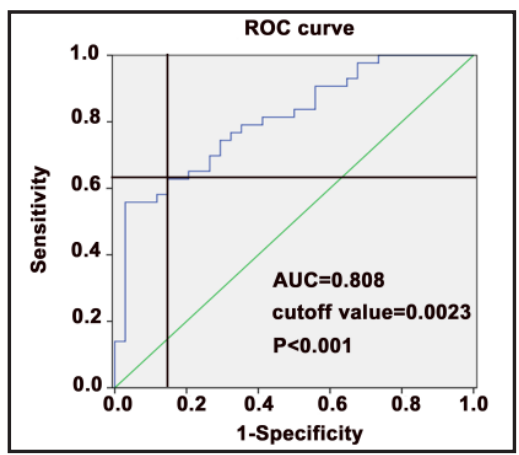


exosomes and unfractionated serum in 10 stable chronic systolic HF patients and 10 controls [14]. They concluded that there was no difference between their levels [14]. Another report showed that extracellular miRNAs were predominantly exosome/microvesicle-free and were associated with Ago proteins [27]. In the present research, we compared the expression of miR-92b-5p between exosomes and exosome-depleted supernatant (Fig. 3), and found that miR-92b-5p was mainly expressed in exosomes, which provides support for the report of Gallo et al. [25].

Before we discuss the main results of this study, we should first discuss one issue that cannot be avoided. What kind of reference should we choose to adjust the results, an internal or external reference? We firstly added cel-miR-39 as an external reference (data not shown) to adjust the final result. We found that although we unified the amount of celmiR-39, cel-miR-39 expression showed significant differences in different experiments. We then compared some internal references according to previous reports, such as miR-16 [2832], U6 [32-34], and miR-451 [21]. We found that miR-451b was stably expressed in serum exosomes without any significant difference between patients with DCM-AHF and the CG (Table 2). Finally, we chose miR-451b as our internal reference for serum exo-miRNAs.

Due to the similarity of its basic sequence, miR-92 can be divided into miR-92a and miR-92b. miR-92a contains 2 members (miR-92a-1 and miR-92a-2) with the same sequence, which are located separately in different miRNA gene clusters. Many changes in the expression of miR-92a have been reported in CVDs. For example, Jiang et al. reported that miR-92a expression was decreased in atherosclerosis [35], whereas it presented with the highest expression level in 95 patients with coronary atherosclerosis [36]. Compared to miR92a, there are much fewer reports on miR-92b in CVDs. One report indicated that miR-92b was expressed higher in CHF patients than in a CG [14]. However, so far as we know, there is no report on the expression of miR-92b in DCM-AHF, and especially no report on serum exomiR-92b levels in DCM-AHF. Our present study showed that exo-miR-92b-5p was increased in patients with DCM-AHF. Given the sequence similarity between miR-92a and miR-92b, we could predict the function of miR-92b by using reports on miR-92a. Animal experiments showed that miR-92a regulated angiogenesis and functional recovery of ischemic tissues in mice [37]. Therefore, patients with DCM-AHF might be in the ischemic tissue condition, which might lead to the increased expression of serum exo-miR-92b-5p. In spite of such an assumption, basic research on the function of exo-miR-92b-5p in patients with DCM-AHF should be conducted in the near future.

miR-192-5p expression has also been reported in many CVDs. It was reported as a p53responsive miRNA [38]. The expression of miR-192 was elevated in patients who developed HF within 1 year after AMI compared with 65 matched controls without a subsequent cardiovascular event after discharge [15]. Fang et al. screened miRNA expression using a human plasma/serum microarray and found that miR-192 was up-regulated in hypertrophic cardiomyopathy [39]. To the best of our knowledge, there is no report on the expression of serum exo-miR-192-5p in DCM-AHF. Our present study showed that there was no significant difference between patients with DCM-AHF and the CG for the expression of exo-miR-1925p (Fig. 2B). We may not be able to compare our results with those from other studies for the following reasons. Firstly, different internal references were used. The reports discussed above used snRNA U6 as an exosomal internal miRNA. However, in the present study, we did not test the stability or expression of snRNA U6, but used exo-miR-451b instead. A different control system might not achieve the same result. Secondly, we focused on a different etiological cause of CVD compared to the previous studies. Reports on HF caused by AMI and hypertrophic cardiomyopathy, as well as DCM-AHF in our present study, might produce different information on the circulatory system. Thirdly, because of a limitation in the number of patients enrolled, we might not have generated a definitive conclusion. If possible, a largescale, multi-center study should be conducted in the near future.

miR-320a is a controversial miRNA in animal models of CVDs. It was reported as a protective miRNA during the process of myocardial ischemia-reperfusion injury in a rat model [40]. It was increased during myocardial ischemia-reperfusion injury, which may 
prevent left ventricular remodeling [40]. While Ren et al. reported that over-expression of miR-320 enhanced cardiomyocyte death and apoptosis in mice undergoing simulated ischemia/reperfusion [41]. They also found that Hsp20, a known cardioprotective protein, was an important candidate target of miR-320 [41]. However, our present study showed that there was no significant difference in miR-320a expression between patients with DCM-AHF and the CG. However, these differences were explained according to our analysis in the above section.

The data shown in Table 1 indicated that serum exo-miR-92b-5p might be affected by other 2 risk factors, namely, diabetes mellitus and renal failure. Therefore, we further analyzed patients with or without the above diseases, and we found that neither diabetes mellitus nor renal failure affected the expression of serum exo-miR-92b-5p. Thus, the differential expression of serum exo-miR-92b-5p might be specific to patients with DCMAHF.

We also analyzed the relationship between exo-miR-92b-5p expression and other ultrasound indices (Fig. 2), because ultrasound indices might be one of the most effective diagnostic factors for DCM patients. We found that exo-miR-92b-5p was positively related to LAD, LVDD, and LVSD, whereas it was negatively related to LVEF and LVFS. It is easy to understand such a relationship. LAD, LVDD, and LVSD represent the enlargement of the cardiac chambers, whereas LVEF and LVFS indicate decreased cardiac function. Both the positive relationship between exo-miR-92b-5p and the enlargement of the cardiac chambers (LAD, LVDD, and LVSD), as well as the negative relationship between exo-miR-92b-5p and the factors representing cardiac functional decline, indicated that exo-miR-92b-5p was a risk factor for patients with DCM-AHF. Considering the cost-effectiveness of diagnosis by ultrasound indices, it is a preferable strategy to use exo-miR-92b as an alternative diagnostic factor for patients with DCM-AHF.

Until now, BNP or N-terminal pro-BNP has been the most useful biomarker for the diagnostic evaluation and risk stratification of patients with AHF [42]. In spite of its widespread use in clinical practice, it is affected by other factors and is not considered a specific biomarker for DCM-AHF. It is better to compare the relationship between exo-miR$92 \mathrm{~b}-5 \mathrm{p}$ and BNP by regression analysis as well as the diagnostic value of BNP by using a ROC curve. However, we could not acquire BNP data from the CG volunteers, because it was beyond our budget to test for BNP and they had no indications for such a test to be performed.

We analyzed the sensitivity and specificity of exo-miR-92b-5p for the diagnosis of DCMAHF by ROC curve analysis (Fig. 6). According to this analysis, although the sensitivity for exo-miR-92b-5p was not sufficiently high, a high specificity was achieved, which might compensate for the low specificity of BNP.

Still, some limitations of this study should be taken into account. Firstly, we only tested 3 different exo-miRNAs as candidate biomarkers, which might not be considered a comprehensive approach. If possible, we should select typical cases to be screened using a microarray, and identify more candidate exo-miRNAs for further sample validation. Secondly, the number of patients enrolled was not large enough. If possible, we should recruit additional patients for a second cohort to validate our findings. Thirdly, the abnormal expression of exo-miRNAs needs to be examined by basic studies in vitro and in vivo. Therefore, if possible, a cellular assay and an animal model should be considered in the near future. Fourthly, it is better to verify the specificity of exo-miR-92b-5p for DCM-AHF by comparing its expression in different tissues, but we were unable to obtain those tissues from the patients with DCMAHF because it required invasive procedures and was ethically restricted.

\section{Conclusion}

Serum exo-miR-92b-5p is a putative specific diagnostic biomarker for patients with DCM-AHF. 


\section{Cellular Physiology Cell Physiol Biochem 2018;46:1939-1950 \begin{tabular}{l|l} 
DOI: 10.1159/000489383 & $\begin{array}{l}\text { O } 2018 \text { The Author(s). Published by S. Karger AG, Basel } \\
\text { www.karger.com/cpb }\end{array}$
\end{tabular}}

Wu et al.: Exo-miR-92b-5p as a Biomarker for DCM-AHF

\section{Acknowledgements}

This study was supported by the Ningbo University Science Foundation (Grant Numbers: XYY15016 and XYY16008) Ningbo, Zhejiang, China.

\section{Disclosure Statement}

The authors declare no conflicts of interest.

\section{References}

1 Aurora P, Edwards LB, Kucheryavaya AY, Christie JD, Dobbels F, Kirk R, Rahmel AO, Stehlik J, Hertz MI: The Registry of the International Society for Heart and Lung Transplantation: thirteenth official pediatric lung and heart-lung transplantation report-2010. J Heart Lung Transplant 2010;29:1129-1141.

- 2 Boon RA, Iekushi K, Lechner S, Seeger T, Fischer A, Heydt S, Kaluza D, Treguer K, Carmona G, Bonauer A, Horrevoets AJ, Didier N, Girmatsion Z, Biliczki P, Ehrlich JR, Katus HA, Muller OJ, Potente M, Zeiher AM, Hermeking H, Dimmeler S: MicroRNA-34a regulates cardiac ageing and function. Nature 2013;495:107110.

3 Hassel D, Cheng P, White MP, Ivey KN, Kroll J, Augustin HG, Katus HA, Stainier DY, Srivastava D: MicroRNA-10 regulates the angiogenic behavior of zebrafish and human endothelial cells by promoting vascular endothelial growth factor signaling. Circ Res 2012;111:1421-1433.

-4 Tsuji M, Kawasaki T, Matsuda T, Arai T, Gojo S, Takeuchi JK: Sexual dimorphisms of mRNA and miRNA in human/murine heart disease. PLoS One 2017;12:e0177988.

-5 van Rooij E, Sutherland LB, Thatcher JE, DiMaio JM, Naseem RH, Marshall WS, Hill JA, Olson EN: Dysregulation of microRNAs after myocardial infarction reveals a role of miR-29 in cardiac fibrosis. Proc Natl Acad Sci U S A 2008;105:13027-13032.

6 Li J, Chan MC, Yu Y, Bei Y, Chen P, Zhou Q, Cheng L, Chen L, Ziegler O, Rowe GC, Das S, Xiao J: miR-29b contributes to multiple types of muscle atrophy. Nat Commun 2017;8:15201.

7 Molina-Navarro MM, Rosello-Lleti E, Ortega A, Tarazon E, Otero M, Martinez-Dolz L, Lago F, GonzalezJuanatey JR, Espana F, Garcia-Pavia P, Montero JA, Portoles M, Rivera M: Differential gene expression of cardiac ion channels in human dilated cardiomyopathy. PLoS One 2013;8:e79792.

8 Mause SF, Weber C: Microparticles: protagonists of a novel communication network for intercellular information exchange. Circ Res 2010;107:1047-1057.

-9 Vickers KC, Remaley AT: Lipid-based carriers of microRNAs and intercellular communication. Curr Opin Lipidol 2012;23:91-97.

10 Buzas EI, Gyorgy B, Nagy G, Falus A, Gay S: Emerging role of extracellular vesicles in inflammatory diseases. Nat Rev Rheumatol 2014;10:356-364.

11 Rink J, Ghigo E, Kalaidzidis Y, Zerial M: Rab conversion as a mechanism of progression from early to late endosomes. Cell 2005;122:735-749.

12 Gupta MK, Halley C, Duan ZH, Lappe J, Viterna J, Jana S, Augoff K, Mohan ML, Vasudevan NT, Na J, SosseyAlaoui K, Liu X, Liu CG, Tang WH, Naga Prasad SV: miRNA-548c: a specific signature in circulating PBMCs from dilated cardiomyopathy patients. J Mol Cell Cardiol 2013;62:131-141.

13 Wang Y, Li M, Xu L, Liu J, Wang D, Li Q, Wang L, Li P, Chen S, Liu T: Expression of Bcl-2 and microRNAs in cardiac tissues of patients with dilated cardiomyopathy. Mol Med Rep 2017;15:359-365.

14 Goren Y, Kushnir M, Zafrir B, Tabak S, Lewis BS, Amir O: Serum levels of microRNAs in patients with heart failure. Eur J Heart Fail 2012;14:147-154.

15 Matsumoto S, Sakata Y, Suna S, Nakatani D, Usami M, Hara M, Kitamura T, Hamasaki T, Nanto S, Kawahara Y, Komuro I: Circulating p53-responsive microRNAs are predictive indicators of heart failure after acute myocardial infarction. Circ Res 2013;113:322-326. 


\section{Cellular Physiology Cell Physiol Biochem 2018;46:1939-1950 \begin{tabular}{l|l} 
DOI: 10.1159/000489383 & O 2018 The Author(s). Published by S. Karger AG, Basel \\
www.karger.com/cpb
\end{tabular}}

Wu et al.: Exo-miR-92b-5p as a Biomarker for DCM-AHF

16 Endo K, Naito Y, Ji X, Nakanishi M, Noguchi T, Goto Y, Nonogi H, Ma X, Weng H, Hirokawa G, Asada T, Kakinoki S, Yamaoka T, Fukushima Y, Iwai N: MicroRNA 210 as a biomarker for congestive heart failure. Biol Pharm Bull 2013;36:48-54.

17 Xu T, Zhou Q Che L, Das S, Wang L, Jiang J, Li G, Xu J, Yao J, Wang H, Dai Y, Xiao J: Circulating miR-21, miR378, and miR-940 increase in response to an acute exhaustive exercise in chronic heart failure patients. Oncotarget 2016; 7:12414-12425.

-18 Richardson P, McKenna W, Bristow M, Maisch B, Mautner B, O’Connell J, Olsen E, Thiene G, Goodwin J, Gyarfas I, Martin I, Nordet P: Report of the 1995 World Health Organization/International Society and Federation of Cardiology Task Force on the Definition and Classification of cardiomyopathies. Circulation 1996;93:841-842.

19 Gheorghiade M, Follath F, Ponikowski P, Barsuk JH, Blair JE, Cleland JG, Dickstein K, Drazner MH, Fonarow GC, Jaarsma T, Jondeau G, Sendon JL, Mebazaa A, Metra M, Nieminen M, Pang PS, Seferovic P, Stevenson LW, van Veldhuisen DJ, Zannad F, Anker SD, Rhodes A, McMurray JJ, Filippatos G: Assessing and grading congestion in acute heart failure: a scientific statement from the acute heart failure committee of the heart failure association of the European Society of Cardiology and endorsed by the European Society of Intensive Care Medicine. Eur J Heart Fail 2010;12:423-433.

20 Mebazaa A: Current ESC/ESICM and ACCF/AHA guidelines for the diagnosis and management of acute heart failure in adults--are there differences? Pol Arch Med Wewn 2009;119:569-573.

-21 Ogata-Kawata H, Izumiya M, Kurioka D, Honma Y, Yamada Y, Furuta K, Gunji T, Ohta H, Okamoto H, Sonoda H, Watanabe M, Nakagama H, Yokota J, Kohno T, Tsuchiya N: Circulating exosomal microRNAs as biomarkers of colon cancer. PLoS One 2014;9:e92921.

-22 Creemers EE, Tijsen AJ, Pinto YM: Circulating microRNAs: novel biomarkers and extracellular communicators in cardiovascular disease? Circ Res 2012;110:483-495.

-23 Fichtlscherer S, Zeiher AM, Dimmeler S: Circulating microRNAs: biomarkers or mediators of cardiovascular diseases? Arterioscler Thromb Vasc Biol 2011;31:2383-2390.

-24 Mitchell PS, Parkin RK, Kroh EM, Fritz BR, Wyman SK, Pogosova-Agadjanyan EL, Peterson A, Noteboom J, O’Briant KC, Allen A, Lin DW, Urban N, Drescher CW, Knudsen BS, Stirewalt DL, Gentleman R, Vessella RL, Nelson PS, Martin DB, Tewari M: Circulating microRNAs as stable blood-based markers for cancer detection. Proc Natl Acad Sci U S A 2008;105:10513-10518.

25 Gallo A, Tandon M, Alevizos I, Illei GG: The majority of microRNAs detectable in serum and saliva is concentrated in exosomes. PLoS One 2012; 7:e30679.

26 Jansen F, Yang X, Proebsting S, Hoelscher M, Przybilla D, Baumann K, Schmitz T, Dolf A, Endl E, Franklin BS, Sinning JM, Vasa-Nicotera M, Nickenig G, Werner N: MicroRNA expression in circulating microvesicles predicts cardiovascular events in patients with coronary artery disease. J Am Heart Assoc 2014;3:e001249.

27 Turchinovich A, Weiz L, Langheinz A, Burwinkel B: Characterization of extracellular circulating microRNA. Nucleic Acids Res 2011;39:7223-7233.

-28 Tokuhisa M, Ichikawa Y, Kosaka N, Ochiya T, Yashiro M, Hirakawa K, Kosaka T, Makino H, Akiyama H, Kunisaki C, Endo I: Exosomal miRNAs from Peritoneum Lavage Fluid as Potential Prognostic Biomarkers of Peritoneal Metastasis in Gastric Cancer. PLoS One 2015;10:e0130472.

29 Yan S, Han B, Gao S, Wang X, Wang Z, Wang F, Zhang J, Xu D, Sun B: Exosome-encapsulated microRNAs as circulating biomarkers for colorectal cancer. Oncotarget 2017; 8:60149-60158.

30 Eichelser C, Stuckrath I, Muller V, Milde-Langosch K, Wikman H, Pantel K, Schwarzenbach H: Increased serum levels of circulating exosomal microRNA-373 in receptor-negative breast cancer patients. Oncotarget 2014;5:9650-9663.

-31 Tanaka Y, Kamohara H, Kinoshita K, Kurashige J, Ishimoto T, Iwatsuki M, Watanabe M, Baba H: Clinical impact of serum exosomal microRNA-21 as a clinical biomarker in human esophageal squamous cell carcinoma. Cancer 2013;119:1159-1167.

-32 Warnecke-Eberz U, Chon SH, Holscher AH, Drebber U, Bollschweiler E: Exosomal onco-miRs from serum of patients with adenocarcinoma of the esophagus: comparison of miRNA profiles of exosomes and matching tumor. Tumour Biol 2015;36:4643-4653.

-33 Wang J, Zhou Y, Lu J, Sun Y, Xiao H, Liu M, Tian L: Combined detection of serum exosomal miR-21 and HOTAIR as diagnostic and prognostic biomarkers for laryngeal squamous cell carcinoma. Med Oncol 2014;31:148. 


\section{Cellular Physiology Cell Physiol Biochem 2018;46:1939-1950

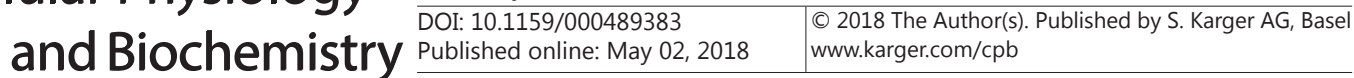

Wu et al.: Exo-miR-92b-5p as a Biomarker for DCM-AHF

-34 Wang H, Hou L, Li A, Duan Y, Gao H, Song X: Expression of serum exosomal microRNA-21 in human hepatocellular carcinoma. Biomed Res Int 2014;2014:864894.

-35 Jiang Y, Wang HY, Li Y, Guo SH, Zhang L, Cai JH: Peripheral blood miRNAs as a biomarker for chronic cardiovascular diseases. Sci Rep 2014;4:5026.

-36 Niculescu LS, Simionescu N, Sanda GM, Carnuta MG, Stancu CS, Popescu AC, Popescu MR, Vlad A, Dimulescu DR, Simionescu M, Sima AV: MiR-486 and miR-92a Identified in Circulating HDL Discriminate between Stable and Vulnerable Coronary Artery Disease Patients. PLoS One 2015;10:e0140958.

-37 Bonauer A, Carmona G, Iwasaki M, Mione M, Koyanagi M, Fischer A, Burchfield J, Fox H, Doebele C, Ohtani K, Chavakis E, Potente M, Tjwa M, Urbich C, Zeiher AM, Dimmeler S: MicroRNA-92a controls angiogenesis and functional recovery of ischemic tissues in mice. Science 2009;324:1710-1713.

-38 Pichiorri F, Suh SS, Rocci A, De Luca L, Taccioli C, Santhanam R, Zhou W, Benson DM, Jr., Hofmainster C, Alder H, Garofalo M, Di Leva G, Volinia S, Lin HJ, Perrotti D, Kuehl M, Aqeilan RI, Palumbo A, Croce CM: Downregulation of p53-inducible microRNAs 192, 194, and 215 impairs the p53/MDM2 autoregulatory loop in multiple myeloma development. Cancer Cell 2010;18:367-381.

-39 Fang L, Ellims AH, Moore XL, White DA, Taylor AJ, Chin-Dusting J, Dart AM: Circulating microRNAs as biomarkers for diffuse myocardial fibrosis in patients with hypertrophic cardiomyopathy. J Transl Med 2015;13:314.

40 Song CL, Liu B, Diao HY, Shi YF, Li YX, Zhang JC, Lu Y, Wang G, Liu J, Yu YP, Guo ZY, Wang JP, Zhao Z, Liu JG, Liu YH, Liu ZX, Cai D, Li Q: The protective effect of microRNA-320 on left ventricular remodeling after myocardial ischemia-reperfusion injury in the rat model. Int J Mol Sci 2014;15:17442-17456.

41 Ren XP, Wu J, Wang X, Sartor MA, Qian J, Jones K, Nicolaou P, Pritchard TJ, Fan GC: MicroRNA-320 is involved in the regulation of cardiac ischemia/reperfusion injury by targeting heat-shock protein 20. Circulation 2009;119:2357-2366.

-42 Yancy CW, Jessup M, Bozkurt B, Butler J, Casey DE, Jr., Drazner MH, Fonarow GC, Geraci SA, Horwich T, Januzzi JL, Johnson MR, Kasper EK, Levy WC, Masoudi FA, McBride PE, McMurray JJ, Mitchell JE, Peterson PN, Riegel B, Sam F, Stevenson LW, Tang WH, Tsai EJ, Wilkoff BL: 2013 ACCF/AHA guideline for the management of heart failure: a report of the American College of Cardiology Foundation/American Heart Association Task Force on practice guidelines. Circulation 2013;128:e240-327. 